\title{
SCHATTEN-TYPE CLASSES ON SEQUENCE SPACES
}

\author{
R. KHALIL AND M. SALEH
}

\begin{abstract}
Let $H$ be a Hilbert space and $L(H)$ be the bounded linear operators on $\mathrm{H}$. For $T \in L(H)$, let $\|T\|_{p}=\sup \left[\sum_{n=1}^{\infty}\left|<T e_{n}, e_{n}>\right|^{p}\right]^{1 / p}$, where the supremum is taken over all orthonormal sequences $\left(e_{n}\right)$. Set $C_{p}(H)=\left\{T \in L(H):\|T\|_{p}<\infty\right\}$. The object of this paper is to define and study $C_{p}(X, Y)$ where $X$ and $Y$ are sequences spaces.
\end{abstract}

\section{Introduction}

Let $H$ be a Hilbert space and $L(H)$ be the space of bounded linear operators on $H$. For $T \in L(H)$ let

$$
\|T\|_{p}=\sup \left[\sum_{n=1}^{\infty}\left|<T e_{n}, e_{n}>\right|^{p}\right]^{1 / p}, 1 \leq p<\infty
$$

where the supremum is taken over all orthonormal sequences $\left(e_{n}\right)$ in H. The Schatten class of index $p$ is defined to be

$$
C_{p}(H)=\left\{T \in L(H):\|T\|_{p}<\infty\right\}
$$

We refer to [4], [5] for more on Schatten classes. The set of compact operators in $L(H)$ are denoted by $C_{\infty}$. It is known that $C_{p}(H) \subset C_{\infty}$, for all $1 \leq p<\infty$, and that $C_{p}(H)$ is a two sided ideal in $L(H)$. Further, for $2 \leq p<\infty$ and $T \in C_{p}(H)$,

$$
\|T\|_{p}=\sup _{\left(e_{n}\right)}\left[\sum_{n=1}^{\infty}\left\|T e_{n}\right\|^{p}\right]^{1 / p} .
$$

And for $1 \leq p \leq 2$,

$$
\|T\|_{p}=\inf _{\left(e_{n}\right)}\left[\sum_{n=1}^{\infty}\left\|T e_{n}\right\|^{p}\right]^{1 / p} .
$$

Schatten classes can be defined on Banach spaces either via singular numbers of bounded operators or via $(p, 2)$-summing operators. We refer to [4] for both cases.

Received December 24, 1996; revised June 10, 1998.

1991 Mathematics Subject Classification. 46E, 46B

Key words and phrases. Schatten classes, sequence spaces. 
The object of this paper is to define Schatten type classes on sequence spaces $\left(\ell^{p}-\right.$ sapces) via $p$-orthogonal sequences.

Throughout this paper $L(E, F)$ is the space of all bounded linear operators from $E$ to $F$, where $E$ and $F$ are any two Banach spaces. the compact operators in $L(E, F)$ will be denoted by $K(E, F)$ while the finite rank operators will be denoted by $F(E, F)$. The class of $(p, q)$-summing operators in $L(E, F)$ is denoted by $\pi_{p, q}(E, F),[4]$, and the class of $p$-nuclear operators will be denoted by $N_{p}(E, F)$, [4]. The class of weakly $p$-summable sequences on $E$ is denoted by $\ell^{p}(E)$, [2]. The dual of $E$ is $E^{*}$, the unit sphere of $E$ is $S(E)$ and the conjugate of $p$ is $p^{*}\left[\frac{1}{p}+\frac{1}{p^{*}}=1\right]$.

I. $C_{p}\left(\ell^{p^{*}}, E\right)$

Let $E$ be any Banach space and $1 \leq p<\infty$. To define our class of operators, we need first to introduce the concept of $p$-orthogonal elements in Banach spaces.

Definiton 1.1. A sequence $\left(x_{n}\right)$ in $E$ is called $p$-orthogonal if

$$
\left\|\sum_{n=1}^{\infty} \lambda_{n} x_{n}\right\|=\left[\sum_{n=1}^{\infty}\left|\lambda_{n}\right|^{P}\left\|x_{n}\right\|^{p}\right]^{1 / p} .
$$

If $\left\|x_{n}\right\|=1$, we say $\left(x_{n}\right)$ is $p$-orthonormal. For $p=\infty,\left(x_{n}\right)$ is called $p$-orthogonal if

$$
\left\|\sum_{n=1}^{\infty} \lambda_{n} x_{n}\right\|=\sup _{n}\left(\left|\lambda_{n}\right|\left\|x_{n}\right\|\right) .
$$

We refer to [1] for more on p-orthogonal sequences in Banach spaces. Some of the basic properties of $p$-orthogonal sequences in listed in:

Lemma 1.2. Let $\left(x_{n}\right)$ be a sequence in the Banach space E. Then:

(i) For $E=\ell^{p},\left(x_{n}\right)$ is p-orthogonal if and only if $\operatorname{supp}\left(x_{n}\right) \cap \operatorname{supp}\left(x_{m}\right)=\varphi$ for $n \neq m$, where $\operatorname{supp}\left(x_{n}\right)=$ closure of $\left\{i: x_{n}(i) \neq 0\right\}$.

(ii) For $E=\ell^{p},\left(x_{n}\right)$ is p-orthogonal if and only if $\left(\left|x_{n}\right|\right)$ is p-orthogonal.

(iii) If $\left(x_{n}\right)$ is p-orthonormal in $E$, then

$$
\left[\sum_{n=1}^{\infty}\left|<x_{n}, x^{*}>\right|^{p}\right]^{1 / p} \leq\left\|x^{*}\right\|
$$

for all $x^{*} \in E^{*}$.

The proof of (i) can be found in [1], (ii) follows from (i) and (iii) follows from the definition of $p$-orthogonal sequences.

Now we introduce our basic definition. 
Definition 1.3. For $1 \leq p<\infty$ and $E$ any Banach space, set:

$$
C_{p}\left(\ell^{p^{*}}, E\right)=\left\{T \in L\left(\ell^{p^{*}}, E\right): \sup \left[\sum_{n=1}^{\infty}\left\|T \theta_{n}\right\|^{p}\right]^{1 / p}<\infty\right\},
$$

where the supremum is taken over all $p^{*}$-orthonormal sets, $\left(\theta_{n}\right)$, in $\ell^{p^{*}}$. for $T \in C_{p}\left(\ell^{p^{*}}, E\right)$, set:

$$
\|T\|_{p}=\sup \left\{\left[\sum_{n=1}^{\infty}\left\|T \theta_{n}\right\|^{p}\right]^{1 / p}:\left(\theta_{n}\right) \text { is } p^{*} \text {-orthonormal set in } \ell^{p^{*}}\right\} .
$$

For $p=\infty$, we let

$$
C_{\infty}\left(\ell^{1}, E\right)=\left\{T \in L\left(\ell^{1}, E\right): \sup _{n}\left(\left\|T \theta_{n}\right\|\right)<\infty\right\},
$$

where the supremum is taken over all 1 -orthonormal sets, $\left(\theta_{n}\right)$, in $\ell^{1}$. For $T \in C_{\infty}\left(\ell^{1}, E\right)$, set:

$$
\|T\|_{\infty}=\sup \left\{\left\|\left(\left\|T \theta_{n}\right\|\right)\right\|_{\infty},\left(\theta_{n}\right) \text { is 1-orthonormal set in } \ell^{1}\right\} .
$$

Throughout this paper, we write sup to denote that the supremum is taken over all $\left(\theta_{n}\right)_{p}$ $p$-orthonormal sets $\left(\theta_{n}\right)$ in $\ell^{p}$ where $1 \leq p \leq \infty$.

Lemma 1.4. Let $E$ and $F$ be any Banach spaces, and $1 \leq p \leq \infty$. Then:

(i) $\|T\| \leq\|T\|_{p}$ for all $T \in C_{p}\left(\ell^{p^{*}}, E\right)$

(ii) For any $A \in L(E, F)$ and all $T \in C_{p}\left(\ell^{p^{*}}, E\right) A T \in c_{p}\left(\ell^{p^{*}}, F\right)$ and $\|A T\|_{p} \leq\|A\|\|T\|_{p}$.

(iii) $C_{p}\left(\ell^{p^{*}}, E\right)$ is a Banach space

(iv) $C_{\infty}\left(\ell^{1}, E\right)=L\left(\ell^{1}, E\right)$.

Proof. The proof of (i), (ii) and (iv) follows from the definition of \|\|$_{p}$. For (iii) we only prove that $C_{p}\left(\ell^{p^{*}}, E\right)$ is complete. for that, by proposition $4[6, p .116]$ it is enough to prove that if $T_{n} \in C_{p}\left(\ell^{p^{*}}, E\right)$ such that $\sum_{n=1}^{\infty}\left\|T_{n}\right\|_{p}<\infty$, then $\sum_{n=1}^{\infty} T_{n} \in C_{p}\left(\ell^{p^{*}}, E\right)$.

Consider $T=\sum_{n=1}^{\infty} T_{n}$, where $T x=\sum_{n=1}^{\infty} T_{n} x$ for all $x \in \ell^{p^{*}}$. Then:

$$
\|T x\| \leq\|x\| \sum_{n=1}^{\infty}\left\|T_{n}\right\| \leq\|x\| \sum_{n=1}^{\infty}\left\|T_{n}\right\|_{p}<\infty .
$$

Hence, $T \in L\left(\ell^{p^{*}}, E\right)$.

Now, let $\left(\theta_{k}\right)$ be any $p^{*}$-orthonormal set in $\ell^{p^{*}}$. Then

$$
\begin{aligned}
{\left[\sum_{k=1}^{\infty}\left\|\sum_{n=1}^{\infty} T_{n} \theta_{k}\right\|^{p}\right]^{1 / p} } & \leq \sum_{n=1}^{\infty}\left[\sum_{k=1}^{\infty}\left\|T_{n} \theta_{k}\right\|^{p}\right]^{1 / p} \\
& \leq \sum_{n=1}^{\infty} \sup _{\left(\theta_{k}\right)_{p^{*}}}\left[\sum_{k=1}^{\infty}\left\|T_{n} \theta_{k}\right\|^{p}\right]^{1 / p} \\
& \leq \sum_{n=1}^{\infty}\left\|T_{n}\right\|_{p}
\end{aligned}
$$


Lemma 1.5. $F\left(\ell^{p^{*}}, E\right) \subset C_{p}\left(\ell^{p^{*}}, E\right)$. Further for any $x \in \ell^{p}$ and $y \in E$ we have $\|x \otimes y\|_{p}=\|x\|\|y\|$.

Proof. Follows from definition 1.3 and the basic properties of \|\|$_{p}$. We now give a nice characterization of $C_{p}\left(\ell^{p^{*}}, E\right)$.

Theorem 1.6. Let $1<p<\infty$ and $p \neq 2$. Then the following are equivalent:

(i) $T \in C_{p}\left(\ell^{p^{*}}, E\right)$.

(ii) $T=\sum_{n=1}^{\infty} \lambda_{n} \delta_{n} \otimes g_{n}$, where $g_{n} \in E$ with $\left\|g_{n}\right\|=1$. In this case, $\|T\|_{p}=\left\|\left(\lambda_{n}\right)\right\|_{p}$.

Proof. (i) $\rightarrow$ (ii).

Let $T \in c_{p}\left(\ell^{p^{*}}, E\right)$ and $x \in \ell^{p^{*}}$. Since $\delta_{n}$ is a basis for $\ell^{p^{*}}, 1<p<\infty$, then $x=\sum_{n=1}^{\infty}<x, \delta_{n}>\delta_{n}$. This implies that

$$
T x=\sum_{n=1}^{\infty}<x, \delta_{n}>T \delta_{n} .
$$

Thus,

$$
T=\sum_{n=1}^{\infty} \delta_{n} \otimes T \delta_{n}
$$

where the series converges strongly. Hence, $T=\sum_{n=1}^{\infty} \lambda_{n} \delta \otimes g_{n}$ where $\lambda_{n}=\left\|T \delta_{n}\right\|$ and $g_{n}=\frac{T \delta_{n}}{\left\|T \delta_{n}\right\|}$. But since $T \in C_{p}\left(\ell^{p^{*}, E}\right)$, then $\left[\sum_{n=1}^{\infty}\left|\lambda_{n}\right|^{p}\right]^{1 / p}=\left[\sum_{n=1}^{\infty}\left\|T \delta_{n}\right\|^{p}\right]^{1 / p} \leq\|T\|_{p}$. Thus

$$
T=\sum_{n=1}^{\infty} \lambda_{n} \delta_{n} \otimes g_{n}
$$

where $\left(\lambda_{n}\right) \in \ell^{p}$ and $\left\|g_{n}\right\|=1$.

(ii) $\rightarrow$ (i).

Let $T=\sum_{n=1}^{\infty} \lambda_{n} \delta_{n} \otimes g_{n}$, where $\left(\lambda_{n}\right) \in \ell^{p}$ and $g_{n} \in E$ with $\left\|g_{n}\right\|=1$. We claim that $T \in C_{p}\left(\ell^{p^{*}}, E\right)$. First, we prove that $T \in L\left(\ell^{p^{*}}, E\right)$. For this, let $x \in \ell^{p^{*}}$. Then

$$
\begin{aligned}
\|T x\| & \leq\left[\sum_{n=1}^{\infty}\left|\lambda_{n}\right|^{p}\right]^{1 / p} \cdot\left[\sum_{n=1}^{\infty}<\delta_{n}, x>\left.\right|^{p^{*}}\right]^{1 / p^{*}} \\
& \leq\left[\sum_{n=1}^{\infty}\left|\lambda_{n}\right|^{p}\right]^{1 / p} \cdot\|x\| .
\end{aligned}
$$

Thus, $T \in L\left(\ell^{p^{*}}, E\right)$ and $\|T\| \leq\left[\sum_{n=1}^{\infty}\left|\lambda_{n}\right|^{p}\right]^{1 / p}$.

Now, let $\left(\theta_{k}\right)$ be any $p^{*}$-orthonormal set in $\ell^{p^{*}}$. Then

$$
\left[\sum_{k=1}^{\infty}\left\|T \theta_{k}\right\|^{p}\right]^{1 / p} \leq\left[\sum_{k=1}^{\infty}\left[\sum_{n=1}^{\infty}\left|\lambda_{n} \|<\delta_{n}, \theta_{k}>\right|\right]^{p}\right]^{1 / p}
$$




$$
\begin{aligned}
& \leq\left|\sum_{k=1}^{\infty} \sum_{n=1}^{\infty} \eta_{k}\right| \lambda_{n} \|<\delta_{n}, \theta_{k}>\mid \quad\left(\left\|\left(\eta_{k}\right)\right\|_{p^{*}}=1\right) \\
& \leq \sum_{k=1}^{\infty} \sum_{n=1}^{\infty}\left|\eta_{k}\right|\left|\lambda_{n}\right|<\delta_{n}|,| \theta_{k} \mid>.
\end{aligned}
$$

Since $\left|\delta_{n}\right|$ is $p$-orthonormal, then $\left\|\sum_{n=1}^{\infty}\left|\lambda_{n}\left\|\delta_{n} \mid\right\|_{p}=\left[\sum_{n=1}^{\infty}\left|\lambda_{n}\right|^{p}\right]^{1 / p}<\infty\right.\right.$. Thus $\sum_{n=1}^{\infty}\left|\lambda_{n}\right|\left|\delta_{n}\right| \in \ell^{p}$. Consequently, since $\left|\theta_{k}\right| \in \ell^{p^{*}}$, then:

$$
\left[\sum_{k=1}^{\infty}\left\|T \theta_{k}\right\|^{p}\right]^{1 / p} \leq \sum_{k=1}^{\infty}\left|\eta_{k}\right|<\sum_{n=1}^{\infty}\left|\lambda_{n}\right|\left|\delta_{n}\right|,\left|\theta_{k}\right|>
$$

Again, since $\left|\theta_{k}\right|$ is $p$-orthonormal, we get

$$
\left\|\sum_{k=1}^{\infty}\left|\eta_{k}\right|\left|\theta_{k}\right|\right\|_{p^{*}}=\left[\sum_{k=1}^{\infty}\left|\eta_{k}\right|^{p^{*}}\right]^{1 / p^{*}}<\infty
$$

Thus $\sum_{k=1}^{\infty}\left|\eta_{k}\right|\left|\theta_{k}\right| \in \ell^{p^{*}}$. Hence,

$$
\begin{aligned}
{\left[\sum_{k=1}^{\infty}\left\|T \theta_{k}\right\|^{p}\right]^{1 / p} } & \leq\left|<\sum_{n=1}^{\infty}\right| \lambda_{n} \| \delta_{n}\left|, \sum_{k=1}^{\infty}\right| \eta_{k}|| \theta_{k}|>|\left(\text { since } \sum_{n=1}^{\infty}\left|\lambda_{n} \| \delta_{n}\right| \in \ell^{p}\right) \\
& \leq\left\|\sum_{n=1}^{\infty}\left|\lambda_{n}\right||\delta|\right\|_{p} \cdot\left\|\sum_{k=1}^{\infty}\left|\eta_{k}\right|\left|\theta_{k}\right|\right\|_{p^{*}} \\
& \leq\left[\sum_{n=1}^{\infty}\left|\lambda_{n}\right|^{p}\right]^{1 / p} \cdot\left[\sum_{k=1}^{\infty}\left|\eta_{k}\right|^{p^{*}}\right]^{1 / p^{*}} \\
& =\left[\sum_{n=1}^{\infty}\left|\lambda_{n}\right|^{p}\right]^{1 / p} .
\end{aligned}
$$

Since $\left(\theta_{k}\right)$ was arbitrary $p^{*}$-orthonormal sequence, it follows that $T \in C_{p}\left(\ell^{p^{*}}, E\right)$ and $\|T\|_{p} \leq\left[\sum_{n=1}^{\infty}\left|\lambda_{n}\right|^{p}\right]^{1 / p}$. But

$$
\left[\sum_{n=1}^{\infty}\left|\lambda_{n}\right|^{p}\right]^{1 / p}=\left[\sum_{n=1}^{\infty}\left\|T \delta_{n}\right\|^{p}\right]^{1 / p} \leq\|T\|_{p} .
$$

Hence

$$
\|T\|_{p}=\left[\sum_{n=1}^{\infty}\left|\lambda_{n}\right|^{p}\right]^{1 / p}
$$

If $p=1$, then we have:

Lemma 1.7. If $T=\sum_{n=1}^{\infty} \lambda_{n} \delta_{n} \otimes g_{n}$, where $\left(\lambda_{n}\right) \in \ell^{1}$ and $g_{n} \in E$ with $\mid g_{n} \|=1$, then $T \in C_{1}\left(\ell^{\infty}, E\right)$. In this case, $\|T\|_{1}=\left\|\left(\lambda_{n}\right)\right\|_{1}$. 
Proof. This is just (ii) $\rightarrow$ (i) in Theorem 1.6.

Let $N_{p, q, r}(X, Y)$ denote the space of $(p, q, r)$ uclear operators, [4,Definition 18.1.1], from $X$ into $Y$. Using $N_{p, q, r}(X, Y)$ we give orther characterization of $C_{p}\left(\ell^{p^{*}}, E\right)$.

Theorem 1.8. Let $1<p<\infty, p \neq 2$, and $E$ be any Banach space. Then the following are equivalent:

(i) $T \in C_{p}\left(\ell^{p^{*}}, E\right)$

(ii) $T \in N_{p, 1, p}\left(\ell^{p^{*}}, E\right)$ and $T=\sum_{n=1}^{\infty} \lambda_{n} x_{n} \otimes y_{n}$, where $\left(\lambda_{n}\right) \in \ell^{p},\left(x_{n}\right)=\left(\left|x_{n}\right|\right) \in \ell^{p^{*}}\left(\ell^{p}\right)$ and $\sup _{n}|| y_{n} \mid<\infty$.

Proof. (i) $\rightarrow$ (ii):

Let $T \in C_{p}\left(\ell^{p^{*}}, E\right)$. Then by Theorem 1.6, $T=\sum_{n=1}^{\infty} \lambda_{n} \delta_{n} \otimes g_{n}$ where $\left(\lambda_{n}\right) \in \ell^{p}$, $\delta_{n}=\left|\delta_{n}\right| \in \ell^{p}$ and $\left\|g_{n}\right\|=1$. Since $\left(\lambda_{n}\right) \in \ell^{p}, \sup _{\left\|x^{*}\right\| \leq 1}\left[\sum_{n=1}^{\infty} \mid<\delta_{n}, x^{* \mid p^{*}}\right]^{1 / p^{*}} \leq 1$ and $\sup _{n}\left\|g_{n}\right\|=1$, then it follows from Definition 18.1.1, [4], that $T \in N_{p, 1, p}\left(\ell^{p^{*}}, E\right)$. Further,

$$
\begin{aligned}
\|T\|_{p, 1, p} & \leq\left[\sum_{n=1}^{\infty}\left|\lambda_{n}\right|^{p}\right]^{1 / p} \cdot \sup _{\left\|x^{*}\right\| \leq 1}\left[\sum_{n=1}^{\infty}\left|<\delta_{n}, x^{*}>\right|^{p^{*}}\right]^{1 / p^{*}} \cdot \sup _{n}\left\|g_{n}\right\| \\
& \leq\left[\sum_{n=1}^{\infty}\left|\lambda_{n}\right|^{p}\right]^{1 / p} \\
& =\|T\|_{p} .
\end{aligned}
$$

(ii) $\rightarrow$ (i).

Let $T \in N_{p, 1, p}\left(\ell^{p^{*}}, E\right)$ such that $T=\sum_{i=1}^{\infty} \lambda_{i} x_{i} \otimes y_{i}$ where $\left(\lambda_{i}\right) \in \ell^{p},\left(x_{i}\right)=\left(\left|x_{i}\right|\right) \in$ $\ell^{p^{*}}\left(\ell^{p}\right)$ and $\sup _{i}\left\|y_{i}\right\|<\infty$. Let $\left(\delta_{k}\right)$ be an $p^{*}$-orthonormal set in $\ell^{p^{*}}$. Then

$$
\begin{aligned}
{\left[\sum_{k=1}^{\infty}\left\|T \delta_{k}\right\|^{p}\right]^{1 / p} } & \leq\left[\sum_{k=1}^{\infty}\left|\sum_{n=1}^{\infty}\right| \lambda_{n}\left\|<x_{n}, \delta_{k}>\left|\left\|y_{n}\right\|\right|^{p}\right]^{1 / p}\right. \\
& \leq\left|\sum_{k=1}^{\infty} \sum_{n=1}^{\infty} \eta-k\right| \lambda_{n}\left\|<x_{n}, \delta_{k}>\right\|\left|y_{n} \|\right|\left(\text { For some }\left(\eta_{k}\right) \in S_{1}\left(\ell^{p^{*}}\right)\right) \\
& \leq\left|\sum_{k=1}^{\infty} \sum_{n=1}^{\infty} \eta_{k}\right| \lambda_{n}|<| x_{n}|,| \delta_{k}\left|>\left\|y_{n}\right\|\right| . \\
& \leq\left|<\sum_{n=1}^{\infty}\right| \lambda_{n}|| x_{n}\left|\left\|y_{n}\right\|, \sum_{k=1}^{\infty} \eta_{k}\right| \delta_{k}|>| \\
& \leq\left\|\sum _ { n = 1 } ^ { \infty } \left|\lambda_{n}\left\|x_{n}\left|\left\|y_{n}\right\|\left\|\left.\right|_{p}\right\| \sum_{k=1}^{\infty} \eta_{k}\right| \delta_{k} \mid\right\|_{p^{*}}\right.\right. \\
& \leq \sup _{n}\left\|y_{n}\right\| \cdot\left[\sum_{n=1}^{\infty}\left|\lambda_{n}\right|^{p}\right]^{1 / p}\left[\sum_{n=1}^{\infty}\left|<x_{n}, x^{*}>\right|^{p^{*}}\right]^{1 / p^{*}}
\end{aligned}
$$


Thus, by Theorem 1.6,

$$
\|T\|_{p} \leq\left[\sum_{n=1}^{\infty}\left|\lambda_{n}\right|^{p}\right]^{1 / p} \cdot \sup _{\left\|x^{*}\right\| \leq 1}\left[\sum_{n=1}^{\infty}\left|<x_{n}, x^{*}>\right|^{p^{*}}\right]^{1 / p^{*}} \cdot \sup _{n}\left\|y_{n}\right\| .
$$

Hence $T \in C_{p}\left(\ell^{p^{*}}, E\right)$.

\section{Ideal Realation of $C_{p}\left(\ell^{p^{*}}, E\right)$}

The proof of the following is immediate and will be omitted:

Theorem 2.1.

(i) $C_{p}\left(\ell^{p^{*}}, E\right) \subseteq K\left(\ell^{p^{*}}, E\right)$

(ii) $\pi_{p}\left(\ell^{p^{*}}, E\right) \subseteq C_{p}\left(\ell^{p^{*}}, E\right)$

(iii) $N_{p}\left(\ell^{p^{*}}, E\right) \subseteq C_{p}\left(\ell^{p^{*}}, E\right)$.

For $p=2$, we have the following nice result:

Theorem 2.2. Let $E$ be any Banach space. Then $C_{2}\left(\ell^{2}, E\right)=\pi_{2}\left(\ell^{2}, E\right)$.

Proof. By Theorem 2.1(ii), we have $\pi_{2}\left(\ell^{2}, E\right) \subseteq C_{2}\left(\ell^{2}, E\right)$.

To prove the other inclusion, let $T \in C_{2}\left(\ell^{2}, E\right)$ and $\left(x_{n}\right)$ be any sequence in $\ell^{2}$. If $\sup _{\left\|x^{*}\right\| \leq 1}\left[\sum_{n=1}^{\infty}\left|<x_{n}, x^{*}>\right|^{2}\right]^{1 / 2}=\infty$, then we have:

$$
\left[\sum_{n=1}^{\infty}\left\|T x_{n}\right\|^{2}\right]^{1 / 2} \leq \infty=\sup _{\left\|x^{*}\right\| \leq 1}\left[\sum_{n=1}^{\infty}<x_{n}, x^{*}>\left.\right|^{2}\right]^{1 / 2}
$$

and $T \in \pi_{2}\left(\ell^{2}, E\right)$.

Assume that $\sup _{\left\|x^{*}\right\| \leq 1}\left[\sum_{n=1}^{\infty}\left|<x_{n}, x^{*}>\right|^{2}\right]^{1 / 2}<\infty$. Define

$$
\begin{aligned}
& A: \ell^{2} \rightarrow \ell^{2} \\
& A=\sum_{n=1}^{\infty} \delta_{n} \otimes x_{\text {船. }} .
\end{aligned}
$$

The for each $x \in \ell^{2}$,

$$
\begin{aligned}
\|A x\| & =\left|\sum_{n=1}^{\infty}<\delta_{n}, x><x_{n}, x^{*}>\right| \quad\left(\text { for some } x^{*} \in S_{1}\left(\ell^{2}\right)\right) \\
& \leq\|x\| \cdot\left[\sum_{n=1}^{\infty}\left|<x_{n}, x^{*}>\right|^{2}\right]^{1 / 2} .
\end{aligned}
$$

Consequently, $A \in L\left(\ell^{2}, \ell^{2}\right)$ and $\|A\| \leq\left[\sum_{n=1}^{\infty}\left|<x_{n}, x^{*}>\right|^{2}\right]^{1 / 2}$. Hence

$$
\left[\sum_{n=1}^{\infty}\left\|T x_{n}\right\|^{2}\right]^{1 / 2}=\left[\sum_{n=1}^{\infty}\left\|T A \delta_{n}\right\|^{2}\right]^{1 / 2}=\|A\|\left[\sum_{n=1}^{\infty}\left\|T \frac{A}{\|A\|} \delta_{n}\right\|^{2}\right]^{1 / 2} .
$$


Set $\tilde{A}=\frac{A}{\|A\|}$. Then $\|\tilde{A}\|=1$. Lemma 2.2 .3 , [5], implies that $\tilde{A}=\sum_{i=1}^{4} \alpha_{i} u_{i}$, where $u_{i}$ 's are unitary operators and $\sum_{i=1}^{4}\left|\alpha_{i}\right|=1$. Thus

$$
\begin{aligned}
{\left[\sum_{n=1}^{\infty}\left\|T x_{n}\right\|^{2}\right]^{1 / 2} } & \leq\|A\|\left[\sum_{n=1}^{\infty}\left[\sum_{i=1}^{4}\left|\alpha_{i}\right|\left\|T u_{i} \delta_{n}\right\|\right]^{2}\right]^{1 / 2} \\
& \leq\|A\|\left[\sum_{i=1}^{4}\left|\alpha_{i}\right|\left[\sum_{n=1}^{\infty}\left\|T u_{i} \delta_{n}\right\|^{2}\right]^{1 / 2}\right] \\
& \leq\|A\|\|T\|_{2},
\end{aligned}
$$

noting that a unitary operator maps orthonormal sets to orthonormal sets. Consequently,

$$
\left[\sum_{n=1}^{\infty}\left\|T x_{n}\right\|^{2}\right]^{1 / 2} \leq\|T\|_{2} \cdot \sup _{\left\|x^{*}\right\| \leq 1}\left[\sum_{n=1}^{\infty}\left|<x_{n}, x^{*}>\right|^{2}\right]^{1 / 2} .
$$

Thus, $T \in \pi_{2}\left(\ell^{2}, E\right)$ and $\|T\|_{\pi_{2}} \leq\|T\|_{2}$.

III. Duality in $C_{p}\left(\ell^{p^{*}}, E\right)$

Theorem 3.1. let $E$ be a reflexive Banach space. Then

(i) $\left[C_{2}\left(\ell^{2}, E\right)\right]^{*}$ is isometrically isomorphic to $\pi_{2}\left(E, \ell^{2}\right)$.

(ii) $\left[C_{p}\left(\ell^{p^{*}}, E\right)\right]^{*}$ is isometrically isomorphic to $C_{p^{*}}\left(\ell^{p}, E^{*}\right), 1<p<\infty$ and $p \neq 2$.

\section{Proof.}

(i) Follows from Theorem 2.2 and the fact that $\left[\pi_{2}\left(\ell^{2}, E\right)\right]^{*}=\pi_{2}\left(E, \ell^{2}\right),[4$, p.296].

(ii) For $A \in C_{p^{*}}\left(\ell^{p}, E^{*}\right)$, define

$$
\begin{aligned}
& F_{A}: C_{p}\left(\ell^{p^{*}}, E\right) \rightarrow \mathbb{C} \\
& F_{A}(T)=\sum_{n=1}^{\infty}<A \delta_{n}, T \delta_{n}>.
\end{aligned}
$$

Then

$$
\begin{aligned}
\left|F_{A}(T)\right| & \leq\left[\sum_{n=1}^{\infty}\left\|A \delta_{n}\right\|^{p^{*}}\right]^{1 / p^{*}} \cdot\left[\sum_{n=1}^{\infty}\left\|T \delta_{n}\right\|^{p}\right]^{1 / p} \\
& \leq\|A\|_{p^{*}} \cdot\|T\|_{p} .
\end{aligned}
$$

Hence, $\left\|F_{A}\right\| \leq\|A\|_{p^{*}}$. This implies that $F_{A}$ is a bounded linear functional on $C_{p}\left(\ell^{p^{*}}, E\right)$.

Further

$$
\begin{aligned}
\|A\|_{p^{*}} & =\left[\sum_{n=1}^{\infty}\left\|A \delta_{n}\right\|^{p^{*}}\right]^{1 / p^{*}} \\
& =\left|\sum_{n=1}^{\infty}<A \delta_{n}, y_{n}>\right| \quad\left(\left\|\left(\left\|y_{n}\right\|\right)\right\|_{p}=1\right)
\end{aligned}
$$


Now, define

$$
\begin{aligned}
& T_{0}: \ell^{p^{*}} \rightarrow E, \\
& T_{0}=\sum_{n=1}^{\infty} \delta_{n} \otimes y_{n} .
\end{aligned}
$$

Then

$$
\left\|T_{0} x\right\| \leq\left[\sum_{n=1}^{\infty}\left|<\delta_{n}, x>\right|^{p^{*}}\right]^{1 / p^{*}} \cdot\left[\sum_{n=1}^{\infty}\left\|y_{n}\right\|^{p}\right]^{1 / p} \leq\|x\| .
$$

Hence, $T_{0} \in L\left(\ell^{p^{*}}, E\right)$. But

$$
\left[\sum_{n=1}^{\infty}\left\|T_{0} \delta_{n}\right\|^{p}\right]^{1 / p}=\left[\sum_{n=1}^{\infty}\left\|y_{n}\right\|^{p}\right]^{1 / p}=1
$$

Then by Theorem 1.6, $T_{0} \in C_{p}\left(\ell^{p^{*}}, E\right)$ and $\left\|T_{0}\right\|_{p}=1$. Thus

$$
\|A\|_{p^{*}}=\left|\sum_{n=1}^{\infty}<A \delta_{n}, T_{0} \delta_{n}>\right|=\left|F_{A}\left(T_{0}\right)\right| \leq\left\|F_{A}\right\| \cdot\left\|T_{0}\right\|_{p}=\left\|F_{A}\right\| .
$$

This implies that $\left\|F_{A}\right\|=\|A\|_{p^{*}}$.

Now, define:

$$
\begin{aligned}
& J: C_{p^{*}}\left(\ell^{p}, E^{*}\right) \rightarrow\left[c_{p}\left(\ell^{p^{*}}, E\right)\right]^{*}, \\
& J(A)=F_{A} .
\end{aligned}
$$

Since $\left\|F_{A}\right\|=\|A\|_{p^{*}}$, it follows that $J$ is an isometry.

We claim that $J$ is onto. To see, let $F \in\left[C_{p}\left(\ell^{p^{*}}, E\right)\right]^{*}$. Define a map

$$
\begin{aligned}
& A: \ell^{p} \rightarrow E^{*} \\
& <A x, y>=F(x \otimes y) .
\end{aligned}
$$

As $x \otimes y \in C_{p}\left(\ell^{p^{*}}, E\right)$, it follows that

$$
|\langle A x, y\rangle| \leq\|F\| \cdot\|x \otimes y\|_{p}=\|F\| \cdot\|x\|\|y\| .
$$

Hence $\|A\| \leq\|F\|$ and $A \in L\left(\ell^{p}, E^{*}\right)$. If $\left(\delta_{n}\right)$ is the $p$-orthonormal basis in $\ell^{p}$, then

$$
\begin{aligned}
{\left[\sum_{n=1}^{\infty}\left\|A \delta_{n}\right\|^{p^{*}}\right]^{1 / p^{*}} } & =\left|\sum_{n=1}^{\infty}<A \delta_{n}, g_{n}>\right| \quad\left(\left\|\left(\left\|g_{n}\right\|\right)\right\|_{p}=1\right) . \\
& =\left|\sum_{n=1}^{\infty} F\left(\delta_{n} \otimes g_{n}\right)\right| .
\end{aligned}
$$


Theorem 1.6 implies that $\sum_{n=1}^{\infty} \delta_{n} \otimes g_{n} \in C_{p}\left(\ell^{p^{*}}, E\right)$ and $\left\|\sum_{n=1}^{\infty} \delta_{n} \otimes g_{n}\right\|_{p}=\left[\sum_{n=1}^{\infty}\left\|g_{n}\right\|^{p}\right]^{1 / p}$. Thus

$$
\begin{aligned}
{\left[\sum_{n=1}^{\infty}\left\|A \delta_{n}\right\|^{p^{*}}\right]^{1 / p^{*}} } & =\left|F\left[\sum_{n=1}^{\infty} \delta_{n} \otimes g_{n}\right]\right| \\
& \leq\|F\| \cdot\left\|\sum_{n=1}^{\infty} \delta_{n} \otimes g_{n}\right\|_{p} \\
& =\|F\| .
\end{aligned}
$$

Theorem 1.6 now implies that $A \in C_{p^{*}}\left(\ell^{p}, E^{*}\right)$.

Now, let $T \in C_{p}\left(\ell^{p^{*}}, E\right)$. Then, by Theorem 2.1, there exists $T_{N}=\sum_{n=1}^{N} \delta_{n} \otimes \delta_{n} \epsilon$ $F\left(\ell^{p^{*}}, E\right)$ such that $\lim _{N \rightarrow \infty}\left\|T_{N}-T^{\prime}\right\|_{p}=0$. Hence

$$
F(T)=\lim _{N \rightarrow \infty} F\left(T_{N}\right)=F_{A}(T) .
$$

Consequently, $F_{A}=F$. This implies that $J$ is an isometric onto operator.

Corollary 3.2. Let $1<p<\infty, p \neq 2$ and $E$ be a reflexive Banach space. Then $C_{p}\left(\ell^{p^{*}}, E\right)$ is reflexive.

\section{Ideal Properties of $C_{p}\left[\ell^{p^{*}}\right]$} [4].

Let $\underset{\sim}{O}$ be an operator ideal [4]. The following definitions are taken from Pietsch

(i) $Q$ is called small if whenever $Q(X, Y)=L(X, Y)$, then $X$ or $Y$ is a finite dimensional space.

(ii) $\underset{\mathcal{Q}}{ }$ is called closed if the closure of $Q(X, Y)$ in $L(X, Y)$ is $(X, Y)$ for all Banach spaces $X$ and $Y$.

(iii) $Q$ is called regular if for all Banach spaces $X$ and $Y, T \in Q(X, Y)$ if and only if $\tilde{k}_{Y} T \in Q\left(X, Y^{* *}\right)$, where $k_{Y}$ is the natural embedding of $Y$ into $Y^{* *}$.

(iv) $Q_{\sim}$ is called injective if whever $J_{Y} T \in \mathcal{Q}\left(X, \ell^{\infty}\left(B_{1}\left(Y^{*}\right)\right)\right)$, then $T \in Q(X, Y)$ for all Banach spaces $X$ and $Y$. Here $J_{Y}$ is the natural embedding of $Y$ into $\ell^{\infty}\left(B_{1}\left(Y^{*}\right)\right)$.

Theorem 4.1. Let $2 \leq p<\infty$. Then $C_{p}\left[\ell^{p^{*}}\right]$ is a small left operator ideal.

Proof. Suppose $C_{p}\left(\ell^{p^{*}}, E\right)=L\left(\ell^{p^{*}}, E\right)$ for some Banach space $E$. Since $\|T\| \leq\|T\|_{p}$, then the identity map I from $C_{p}\left(\ell^{p^{*}}, E\right)$ into $L\left(\ell^{p^{*}}, E\right)$ is a bounded linear operator which is onto. The opern mapping theorem now implies that there exists $\gamma>0$ such that for each $T \in C_{p}\left(\ell^{p^{*}}, E\right),\|T\|_{p} \leq \gamma\|T\|$.

Now, assume if possible that $E$ is infinite dimensional Banach space. Chosse $N$ and $\epsilon>0$ such that $N^{1 / p}>\gamma(1+\epsilon)$. Then by Dvoretzky's Lemma [4, p.39], there exists $\left(x_{i}\right)_{n=1}^{N} \in E$ with $\left\|x_{i}\right\|=1$ such that

$$
\sup _{\left\|x^{*}\right\| \leq 1}\left[\sum_{i=1}^{N}\left|<x_{i}, x^{*}>\right|^{2}\right]^{1 / 2} \leq 1+\epsilon
$$


Define,

$$
\begin{aligned}
& J: \ell^{p^{*}} \rightarrow E \\
& J=\sum_{n=1}^{N} \delta_{i} \otimes x_{i} .
\end{aligned}
$$

Then for each $x \in \ell^{p^{*}}$, we have:

$$
\begin{aligned}
\|J x\| & =\left|\sum_{i=1}^{N}<\delta_{i}, x><x_{i}, x^{*}>\right| \quad\left(\text { For some } x^{*} \in S_{1}\left(E^{*}\right)\right) \\
& \leq\left[\sum_{i=1}^{N}\left|<\delta_{i}, x>\right|^{p^{*}}\right]^{1 / p^{*}} \cdot\left[\sum_{i=1}^{N}\left|<x_{i}, x^{*}>\right| p\right]^{1 / p} \\
& \leq\|x\| \cdot\left[\sum_{i=1}^{N}\left|<x_{i}, x^{*}>\right|^{2}\right]^{1 / 2} \\
& \leq(1+\epsilon)\|x\| \quad(\operatorname{By}(1)) .
\end{aligned}
$$

Thus, $\|J\| \leq(1+\epsilon)$. Further

$$
\|J\|_{p} \geq\left[\sum_{i=1}^{N}\left\|J \delta_{i}\right\|^{p}\right]^{1 / p}=\left[\sum_{i=1}^{N}\left\|x_{i}\right\|^{p}\right]^{1 / p}=N^{1 / p} .
$$

Hence, $\|J\|_{p} \geq N^{1 / p}$. Consequently, $N^{1 / p} \leq\|J\|_{p} \leq \gamma\|J\| \leq \gamma(1+\epsilon)<N^{1 / p}$. This is a contradiction. Hence $E$ must be finite dimensional.

Theorem 4.2. Let $2 \leq p<\infty$. Then $C_{p}\left[\ell^{p^{*}}\right]$ is not closed.

Proof. Suppose $C_{p}\left[\ell^{p^{*}}\right]$ is closed. Then $C_{p}\left(\ell^{p^{*}}, E\right)$ is closed in $L\left(\ell^{p^{*}}, E\right)$ for all Banach spaces $E$. Let $E=\ell^{q}$ where $1<q<p^{*} \leq 2$. lemma 1.4 implies that $F\left(\ell^{p^{*}}, \ell^{q}\right) \subseteq$ $C_{p}\left(\ell^{p^{*}}, \ell^{q}\right)$. Then $\overline{F\left(\ell^{p^{*}}, \ell^{q}\right)} \subseteq C_{p}\left(\ell^{p^{*}}, \ell^{q}\right)$ where the closure is in $L\left(\ell^{p^{*}}, \ell^{q}\right)$. Thus, $K\left(\ell^{p^{*}}, \ell^{q}\right) \subseteq C_{p}\left(\ell^{p^{*}}, \ell^{q}\right),[2, \mathrm{p} .242]$. Hence, by Theorem $2.1, C_{p}\left(\ell^{p^{*}}, \ell^{q}\right)=K\left(\ell^{p^{*}}, \ell^{q}\right)$. Corollary 4.2, [3], now implies that $C_{p}\left(\ell^{p^{*}}\right), \ell^{q}=L\left(\ell^{p^{*}}, \ell^{q}\right)$. This contradicts Theorem 4.2. Hence $C_{p}\left[\ell^{p^{*}}\right]$ is not closed.

Theorem 4.3. Let $1<p<\infty$. Then $C_{p}\left[\ell^{p^{*}}\right]$ is regular.

Proof. Let $E$ be any Banach space and $K_{E}$ be the natural embedding of $E$ into $E^{* *}$. We want to prove that $T \in C_{p}\left(\ell^{p^{*}}, E\right)$ if and only if $K_{E} T \in C_{p}\left(\ell^{p^{*}}, E^{* * *}\right)$.

Let $p=2$ and $T \in C_{2}\left(\ell^{2}, E\right)$. Then by lemma 1.4 , we have $K_{E} T \in C_{2}\left(\ell^{2}, E^{* *}\right)$.

Conversely, suppose $K_{E} T \in C_{2}\left(\ell^{2}, E^{* *}\right)$. Theorem 2.2 implies that $K_{E} T \in \pi_{2}\left(\ell^{2}, E^{* *}\right)$. AS $\pi_{2}$ is a regular operator ideal, [4, p.109], it follows that $T \in \pi_{2}\left(\ell^{2}, E\right)$. Consequently, Theorem 2.2 implies that $T \in C_{2}\left(\ell^{2}, E\right)$. Hence $C_{2}\left[\ell^{2}\right]$ is regular.

For $1<p<\infty$ and $p \neq 2$, let $T \in C_{p}\left(\ell^{p^{*}}, E\right)$. Then Lemma 1.4 implies that $K_{E} T \in C_{p}\left(\ell^{p^{*}}, E^{* *}\right)$. 
Now, suppose $K_{E} T \in C_{p}\left(\ell^{p^{*}}, E^{* *}\right)$. Then, Theorem 1.6 implies that:

$$
K_{E} T=\sum_{n=1}^{\infty} \delta_{n} \otimes K_{E} T \delta_{n},
$$

where $\left[\sum_{n=1}^{\infty}\left\|K_{E} T \delta_{n}\right\|^{p}\right]^{1 / p}<\infty$. Since $K_{E}$ is an isometric operator, then,

$$
\left[\sum_{n=1}^{\infty}\left\|T \delta_{n}\right\|^{p}\right]^{1 / p}<\infty
$$

Thus, by Theorem 1.6, we have $T \in C_{p}\left(\ell^{p^{*}}, E\right)$. Hence, $C_{p}\left[\ell^{p^{*}}\right]$ is regular.

In a similar way one can prove:

Theorem 4.4. Let $1<p<\infty$. Then $C_{p}\left[\ell^{p^{*}}\right]$ is injective.

\section{References}

[1] E. Behrends, $L^{p}$-Structure in Real Banach Spaces, Lecture Notes in Math. 613, SpringerVerlag, New York, 1977.

[2] J. Cohen, Absolutely p-summing, p-nuclear operators and their conjugates, Math. Ann. 201(1973), 177-200.

[3] J. Diestel and Jr. Uhl, Vector Measures, Math. Surveys 15, Amer. Math. Soc., Providence, R. I., 1977.

[4] A. Pietsch, Operator Ideals, North-Holland Publishing Company, Amsterdam, 1980.

[5] J. R. Ringrose, Compact Non-self-adjoint Operators, Van Nostrand Rein Hold Company, London, 1971.

[6] H. L. Royden, Real Analysis, Macmillan Publishing Co., INC. New York, 1968.

[7] J. Diestel, H. Jarchow and A. Tonge, Absolutely Summing Operators, Cambridege Studies in Advanced Mathematics, no. 43, Cambridge University Press. 1995.

Department of Mathematics, University of Jordan, Amman, Jordan. 